\title{
Zwitterionic silicone materials derived from aza-Michael reaction of amino-functional PDMS with acrylic acid
}

\author{
A. Genest,${ }^{1,2}$ D. Portinha, ${ }^{1}$ E. Pouget,${ }^{2}$ K. Lamnawar, ${ }^{l}$ F. Ganachaud, ${ }^{1, *}$ E. Fleury ${ }^{1, *}$ \\ ${ }^{1}$ Université de Lyon, CNRS, UMR 5223, INSA-Lyon, IMP@INSA, F-69621, Villeurbanne, France \\ ${ }^{2}$ Elkem Silicones France, 55 Rue des Frères Perret, BP 22, F-69191 Saint-Fons Cedex, France
}

\begin{abstract}
Supramolecular zwitterionic silicones were synthesized by aza-Michael reaction between acrylic acid and amine-functional polydimethylsiloxanes. The in-depth characterization of this chemistry, applied for the first time to silicones, was investigated first with model alkyl amines (hexylamine, 2-ethylhexylamine and N-propylethylenediamine), a model oligosiloxane (3-aminopropylmethyl bis(trimethylsiloxy)silane) and finally various amino-polysiloxanes. It was shown that after a first acid base reaction resulting in ionic pairing, aza-Michael addition proceeded smoothly in mild conditions $\left(50^{\circ} \mathrm{C}\right.$, 1 week reaction). Acrylic acid does not homopolymerize in these conditions and chemical rearrangement of the siloxane backbone through redistribution is negligible. Both mono-adducts and di-adducts, together with residual amine, were titrated, mainly by NMR. The supramolecular assembly of the thus-created zwitterionic moieties was highlighted by a concomitant increase in viscosity and phaseseparation, as observed by TEM, bringing an additional glass transition at $-40{ }^{\circ} \mathrm{C}$ assigned to highly polar ionic clusters. Below the stoichiometry in acrylic acid, all zwitterionic silicones followed the same classical behavior of non-entangled polymers according to the Rouse model, whereas upon introducing an excess of acrylic acid to amino groups, an enhancement of the elasticity was observed. Finally silicone elastomers with solid-like behavior and elastomeric mechanical properties were obtained using a high molar mass polymer bearing bifunctional $\mathrm{N}$-(2-aminoethyl)-3-aminopropyl units that favored a high degree of physical crosslinking.
\end{abstract}




\section{Introduction}

Supramolecular assemblies of (macro)molecules have been intensively investigated for the past decades, first by Lehn and more recently by many researchers around the world [1]. The introduction of noncovalent intermolecular interactions, i.e. ionic, hydrogen, $\pi-\pi$ stacking and hydrophobic interactions or metal-ligand coordination, render possible the generation of a wide range of supramolecular polymers with original self-assembled structures and hence physical behaviors [2]. Among these, ionic pairing interactions are very attractive since they exhibit binding energies in the range of $50-250 \mathrm{~kJ} \cdot \mathrm{mol}^{-1}$, close to that of covalent bonds $\left(\sim 350 \mathrm{~kJ}^{\mathrm{mol}}{ }^{-1}\right),[3]$ while still being easily reversible even at room temperature. One can thus obtain a great variety of physical and mechanical properties by tuning e.g. the ionic content, the nature of the counter-ion, or the characteristic and the structure of the supramolecular polymer. Another important feature in macromolecular supramolecular assembly is the phase separation that generally occurs between the incompatible clustered ion pairs and the polymer segments.

This immiscibility effect is particularly exacerbated with highly hydrophobic polysiloxane chains. For instance, Klok et al. [4] observed a strong increase of the viscosity of carboxylic acid-substituted poly(dimethylsiloxane)s (PDMS-COOH) when the temperature was raised above $70^{\circ} \mathrm{C}$, because of the segregation of carboxylic groups associated with a LCST behavior. They also prepared silicone elastic networks by mixing PDMS-COOH and zinc acetylacetonate at ambient temperature. The authors described the formation of ion-pair multiplets and clusters, particularly at low temperatures. Similar approaches were adopted by Saxene et al. [5] and Cumming et al. [6] who studied the synthesis and viscoelastic properties of polysiloxanes bearing sodium salts of either sulfonic acid or undecylenic acid groups, respectively. Here again, the addition of cations such as lithium $\left(\mathrm{Li}^{+}\right)$, $\operatorname{zinc}\left(\mathrm{Zn}^{2+}\right)$, or magnesium $\left(\mathrm{Mg}^{2+}\right)$, led to an increase in viscosity and rubbery plateau moduli, the values of which were generally higher with divalent cations. More recently Lu et al.[7] described the construction of supramolecular 
silicone elastomers by titrating poly(aminopropylmethylsiloxane) with organic or inorganic acids. Such protocol, also called 'salt-forming vulcanization', favors aggregation that promotes improved mechanical properties.

Another class of ionic silicone materials based on zwitterionic supramolecular assemblies was first reported by Graiver et al. [8] and Yu et al. [9] in the 70-80's. They performed the quaternization of primary and secondary amines on a functional PDMS by ring-opening addition of $\gamma$-propanesultone, the stoichiometry tuning the final content of mono- and dizwitterionomers. The morphology of these materials was assessed by SAXS and microscopy analyses, highlighting ionic domains whose numbers (but not volumes) depended on the ionic concentration.[10] A particularly striking 1000-fold increase of the elastic modulus was observed for high ionic content polysiloxanes (0.075 MPa for 1 mol-\% versus $75 \mathrm{MPa}$ for 10 mol-\%) correlated with the decrease of the elongation properties (respectively 176 and $50 \%) \cdot[11]$

In the quest for a less toxic, more sustainable and economic protocol, we turned to a simple and attractive strategy that implies aza-Michael addition to obtain new zwitterionic polysiloxanes. This approach entails a reaction between acrylic acid and various aminofunctionalized silicone-based oligomers. All these reagents are industrially available: aminopolysiloxanes are widely used in detergents as softeners [12] whereas acrylic acid is primarily utilized as a starting material in the production of acrylic esters,[13] as a (co)monomer for poly(acrylic acid and salt) (co)polymers used as adsorbents or dispersants. Aza-Michael reactions involving amino-functionalized polydimethylsiloxane are largely described in the literature.[14,15] On the other hand, the aza-Michael reaction of carboxylic acid-functionalized Michael acceptors with amines has barely been studied over the past decades, in part because the first data showed that these acceptors were less reactive than alkyl acrylates, for instance. This was probably due to the formed ammonium salt considerably impairing the nucleophilic properties of the amine.[16] 
Nevertheless, investigations have demonstrated that a direct addition is possible as in the case of the addition of acrylic acid to monoethanolamine performed in the presence of a heterogeneous catalyst such as alumina or bentonite, to selectively form a monoadduct.[17] The reaction of primary and secondary aryl amines has been also investigated with acrylic acid in ethanol and at room temperature. After 24 hours, expected adducts were obtained and the temperature increased up to $65^{\circ} \mathrm{C}$ without evidence of acrylic acid polymerization.[18] Finally, microwave irradiations have been used to perform the Michael addition of adenine to acrylic acid within only 20 minutes, but in the presence of a large amount of 1,4-diazabicyclo[2.2.2] octane (DABCO) and a catalytic amount of tetrabutylammonium bromide (TBAB).[19] In all these articles, peculiar amines (ethanolamine, arylamine, azaheterocyles) were used to perform the aza-Michael reaction with acrylic acid in solution and with catalytic input; bulk reaction with simple alkylamines lacked and were thus performed here.

There are also few examples of addition of acrylic acid to amine-functional polymers. For instance, a $\beta$-alanine functionality was introduced into aminopolystyrene chains by the azaMichael addition of poly(4-aminostyrene) to acrylic acid in order to obtain a chelating resin capable of selectively extracting copper. The reaction was performed in toluene at room temperature or at $60^{\circ} \mathrm{C}$, leading after respectively 20 and 5.5 hours to a similar level of substitution.[20] N-(2-carboxyethyl)chitosan (NCE-chitosan) was also prepared by simply adding acrylic acid to a diluted solutions of chitosan.[21] A way to selectively prepare mono$\mathrm{N}$-(2-carboxyethyl)chitosan has also been described and involved a gel-state synthesis, meaning a high concentration of chitosan in water and in the presence of magnesium halides such as $\mathrm{MgCl}_{2}$. With this approach, acrylic acid in large excess (acrylic acid:chitosan mol ratio of 2) and a temperature of $50^{\circ} \mathrm{C}$ led to a degree of substitution close to 1 after $24 \mathrm{~h}$ of reaction.[22]

The present work aims at studying the synthesis and supramolecular properties of novel zwitterionic silicones obtained from an aza-Michael reaction between acrylic acid and various amine-functional polydimethylsiloxanes. To settle the conditions of such aza-Michael reaction, 
the reaction of acrylic acid with model alkylamines that differs from amines studied in the literature, namely hexylamine, 2-ethylhexylamine and N-propylethylenediamine, is first reported; then, a simple oligosiloxane and two types of silicones derivatives bearing 3aminopropyl (primary amine) and $\mathrm{N}$-(2-aminoethyl)-3-aminopropyl (both primary and secondary amines) groups were put into play and characterized. Finally, rheological and viscoelastic properties of the thus-obtained new materials were investigated and are discussed in light of supramolecular assembly structuration.

\section{Experimental section}

\subsection{Materials}

Unless otherwise stated, all reagents were used as received without further purification. Acrylic acid ( $\geq 99 \%)$ (1), propionic acid (99.5\%) (2), hexylamine (99\%) (3), 2-ethylhexylamine (98\%) (4), N-propylethylenediamine ( $\geq 97 \%$ ) (5) and all solvents were purchased from Aldrich. 3aminopropylmethyl bis(trimethylsiloxy)silane (SIA0604.5) (6) was purchased from Gelest. Fluid NH40D (bis-(3-amino propyl)-terminated PDMS) (7) was kindly supplied by Wacker Chemie. AMS 191 (8), (3-amino propyl-grafted PDMS) and AMS 233 (9), an N-(2-aminoethyl)-3-aminopropylmethylsiloxane-grafted PDMS, were purchased from Gelest; whereas, Extrasoft (10) and Modified H21642 (both N-(2-aminoethyl)-3-aminopropylmethylsiloxane-grafted PDMSs) (11) were kindly provided by Elkem Silicones.

\subsection{Synthesis}

In the following reaction descriptions, the molar ratio of reactive groups $r$ is defined as the ratio of the molar amount of $\mathrm{C}=\mathrm{C}$ bonds of a Michael acceptor to the molar amount of $\mathrm{N}-\mathrm{H}$ bonds of a Michael donor, such that a primary amine contains two $\mathrm{N}-\mathrm{H}$ bonds.

$$
r=\left(n_{\mathrm{C}=\mathrm{C}} / n_{\mathrm{N}-\mathrm{H}}\right)
$$




\subsubsection{Aza-Michael reaction with alkylamines $(r=0.5)$ :}

In a typical recipe, acrylic acid (1) (3.6g $50 \mathrm{mmol})$ was slowly added to hexylamine (3) (5.2g, $50 \mathrm{mmol}$ ) in an ice-bath (exothermic reaction) and the homogeneous mixture was stirred at $50^{\circ} \mathrm{C}$ until completion of the reaction, which was monitored by ${ }^{1} \mathrm{H}$ NMR. The final products were analyzed by 1D, 2D NMR and electrospray ionization mass spectroscopies. Same protocol was applied to the two other organic (di)amines.

\subsubsection{Aza-Michael reaction with amine-functional siloxane oligomers or polymers $(r=0.5)$ :}

As an example, acrylic acid (1) $(0.14 \mathrm{~g}, 2.0 \mathrm{mmol})$ was slowly added to bis-(3-aminopropyl)terminated PDMS (7) $(2.9 \mathrm{~g}, 1.0 \mathrm{mmol})$ in an ice-bath (exothermic reaction) and the homogeneous mixture was stirred at $50^{\circ} \mathrm{C}$ until completion of the reaction, which was monitored by ${ }^{1} \mathrm{H}$ NMR. The final products were analyzed by 1D NMR. Same protocol was used for the different amine-functional siloxane polymers. Conversion in acrylic acid for all experiments reported in this study are given in SI.1.

\subsection{Analytical techniques}

\subsubsection{Chemical and physical characterizations.}

${ }^{1} \mathrm{H}$ nuclear magnetic resonance (NMR) spectra were recorded on a Bruker Avance II and Avance III apparatus at respectively 250 or $400 \mathrm{MHz}$ while ${ }^{13} \mathrm{C}$ and ${ }^{29} \mathrm{Si}$ NMR spectra were obtained at respectively 100 and $79.5 \mathrm{MHz}$ on a Bruker Avance III apparatus. The samples were dissolved in $\mathrm{CDCl}_{3}$ at $27^{\circ} \mathrm{C}$ and chemical shifts for ${ }^{1} \mathrm{H}$ NMR were determined in ppm downfield relative to the residual $\mathrm{CHCl}_{3}$ at $\delta_{\mathrm{H}}=7.26 \mathrm{ppm}$. Chemical shifts for ${ }^{13} \mathrm{C}$ NMR were quoted as ppm relative to the central peak of the deuterochloroform triplet $\left(\delta_{\mathrm{C}}=77.16 \mathrm{ppm}\right)$ as the internal standard. For Michael products derived from alkylamines, either a deuterated solvent mixture composed of $\mathrm{CDCl}_{3} / \mathrm{MeOD}\left(75 / 25\right.$ mol-\%) or $\mathrm{D}_{2} \mathrm{O}$ was used. In the latter, 
chemical shifts were reported in ppm relative to TSPd4 (sodium tetra deuterated trimethylsilyl propionate) as the external standard.

Mass spectra were recorded in a positive ion mode on a hybrid quadrupole time-of-flight mass spectrometer (MicroTOFQ-II, Bruker Daltonics, Bremen, Germany) with an Electrospray Ionization (ESI) source between 50 and $1500 \mathrm{~m} / \mathrm{z}$ with a capillary voltage set at $+3,5 \mathrm{kV}$. The solutions were infused directly into the mass spectrometer at $180 \mu \mathrm{L} / \mathrm{h}$ and the calibration was done with sodium formate.

DSC experiments (Q20, TA Instrument with a liquid nitrogen cooling system (LNCS)) were performed with two cycles divided into two steps: a heating ramp from $-150^{\circ} \mathrm{C}$ to $100^{\circ} \mathrm{C}$ followed by a cooling ramp from $100^{\circ} \mathrm{C}$ to $-150^{\circ} \mathrm{C}$. The first cycle is usually performed to erase the thermal history of the sample, whereas the second cycle enables the precise measurement of the different thermal transitions.

TGA experiments were performed under nitrogen at a heating rate of $10^{\circ} \mathrm{C} / \mathrm{min}$ from room temperature to $1000^{\circ} \mathrm{C}$ with a Q500 apparatus from TA Instrument.

\subsubsection{Steady flow and Small Amplitude Oscillatory Shear (SAOS) Rheology.}

Firstly, viscosity measurements were carried out in steady flow regime using a stress-controlled rotational rheometer (TA-DHRII) at $25^{\circ} \mathrm{C}$ with a cone/plate geometry (Cone angle $=1.994^{\circ}$, $\emptyset=40 \mathrm{~mm}$, gap $=52 \mu \mathrm{m})$. Shear stress as well as normal stress difference and viscosity were evaluated by increasing the shear rate from 0.01 to $100 \mathrm{~s}^{-1}$ in the experimental confident window before an edge failure limit.

Secondly, the linear viscoelastic properties of the all the prepared specimens were studied at $25^{\circ} \mathrm{C}$ using the same stress-controlled rotational rheometer (TA-DHRII) but with a parallelplate geometry $(40 \mathrm{~mm}$ in diameter). To ensure that all the SAOS measurements were performed within the linear viscoelastic regime, a dynamic strain sweep test was primarily conducted in a strain amplitude range from 0.01 to $100 \%$ with a maximum frequency of $100 \mathrm{~Hz}$. 
Throughout the measurement, it was verified that the axial force was always equal to zero and that the gap of ca. $1.2 \mathrm{~mm}$ was well controlled within $0.1 \mu \mathrm{m}$ of deviation. Then, dynamic frequency sweep tests were carried out under fixed strain amplitude of 5\%, within the linear viscoelastic region at frequencies from 0.01 to $100 \mathrm{~Hz}$. For zwitterionic silicones, the frequency range was set from 0.01 to $15 \mathrm{~Hz}$.

\section{Results and Discussion}

\subsection{Reactions with model alkylamines}

The aza-Michael reaction involves an alkene molecule and either a primary or a secondary amine as a nucleophile; as a consequence, a primary amine used as a donor can undergo two consecutive Michael additions, such that a mixture of primary, secondary, mono-adduct (MA) and tertiary di-adduct (DA) amines may result [23]. In a previous paper [15], we reported a full study of the impact of operation conditions (temperature, solvent, reactant stoichiometry) on the kinetics and selectivity (MA vs DA). To improve both factors, both protic polar solvent (alkyl alcohol (MA) or fluoro alcohol (DA) or mixture of them) and an excess of Michael acceptors (slight (MA) or large (DA) excess) were necessary. Kinetics were also accelerated by increasing the temperature. Our initial intention here was to prepare highly viscous and elastomeric-type materials, so that removing solvents or excess reagent at the end of the process would not be relevant. Thus, only temperature has been tuned in this study but not to a large extent either, to avoid any possible side reaction such as amidation.

Thus, the aza-Michael reaction of acrylic acid (1) with hexylamine (3) and 2ethylhexylamine (4) was first performed at a ratio $r=0.5$ without any solvent or catalyst ( $r$ is defined as the ratio of the molar amount of $\mathrm{C}=\mathrm{C}$ bonds of a Michael acceptor to the molar amount of N-H bonds of a Michael donor). At the start of the addition of acrylic acid to the amine without heating, the acid-base reaction occurred instantaneously with a sharp heat release (up to $70^{\circ} \mathrm{C}$ ) relative to the large difference of $\mathrm{pK}_{\mathrm{a}}$ between acid and amine groups (SI.2). 
Subsequently, the reactional medium was kept at $50^{\circ} \mathrm{C}$ during 120 hours to let the aza-Michael reaction proceed (Figure 1A). The products were analyzed by ${ }^{1} \mathrm{H},{ }^{13} \mathrm{C}$ and $2 \mathrm{D}-\mathrm{NMR}$, namely COSY, HMBC, and HSQC (SI.3 for the reaction of hexylamine (3) with acrylic acid (1) and SI.4 for the reaction of 2-ethylhexylamine (4) with acrylic acid (1)). An example of ${ }^{1} \mathrm{H}$ NMR and assigned structures of the Michael adducts/reagent is presented in Figure $\mathbf{2}$ for the reaction of hexylamine (3) with acrylic acid (1). All peaks were successfully ascribed both in ${ }^{1} \mathrm{H}$ and ${ }^{13} \mathrm{C}$ NMR spectra.

The Michael reaction was considered complete when the vinylic protons of acrylic acid showing up at 5.5-6.5 ppm had almost entirely vanished and when appeared signals at 2.7-3.0 ppm corresponding to the well-defined and separated peaks of the mono- and di-adducts protons of a methylene group in the $\alpha$ position to the secondary (peaks $f$ and $g$ ) and tertiary amine (peaks $j$ and $k$ ) functions. No peak attesting to the polymerization of acrylic acid was detected and only a small amount (1-2 mol\%) of Michael products arising from 2-carboxyethylacrylate impurities were observed (see SI.3). The composition of Michael adducts was determined from integral values of peaks $d, f$, and $j$ (see SI.3) and reported in Table 1.

Table 1: Composition of reactional mixture obtained from the aza-Michael reaction of acrylic acid (1) with primary alkylamines $\left(50^{\circ} \mathrm{C}, 120 \mathrm{~h}, r=0.5\right)$

\begin{tabular}{cccc}
\hline Reaction based on & \multicolumn{3}{c}{ Composition (mol-\%) } \\
\cline { 2 - 4 } & Primary amine & Mono-adduct & Di-adduct \\
\hline Hexylamine (3) & (3) $37 \pm 1$ & (3MA) $35 \pm 1$ & (3DA) $29 \pm 0$ \\
\hline 2-ethylhexylamine (4) & (4) $32 \pm 1$ & (4MA) $44 \pm 2$ & (4DA) $24 \pm 1$ \\
\hline
\end{tabular}


A)

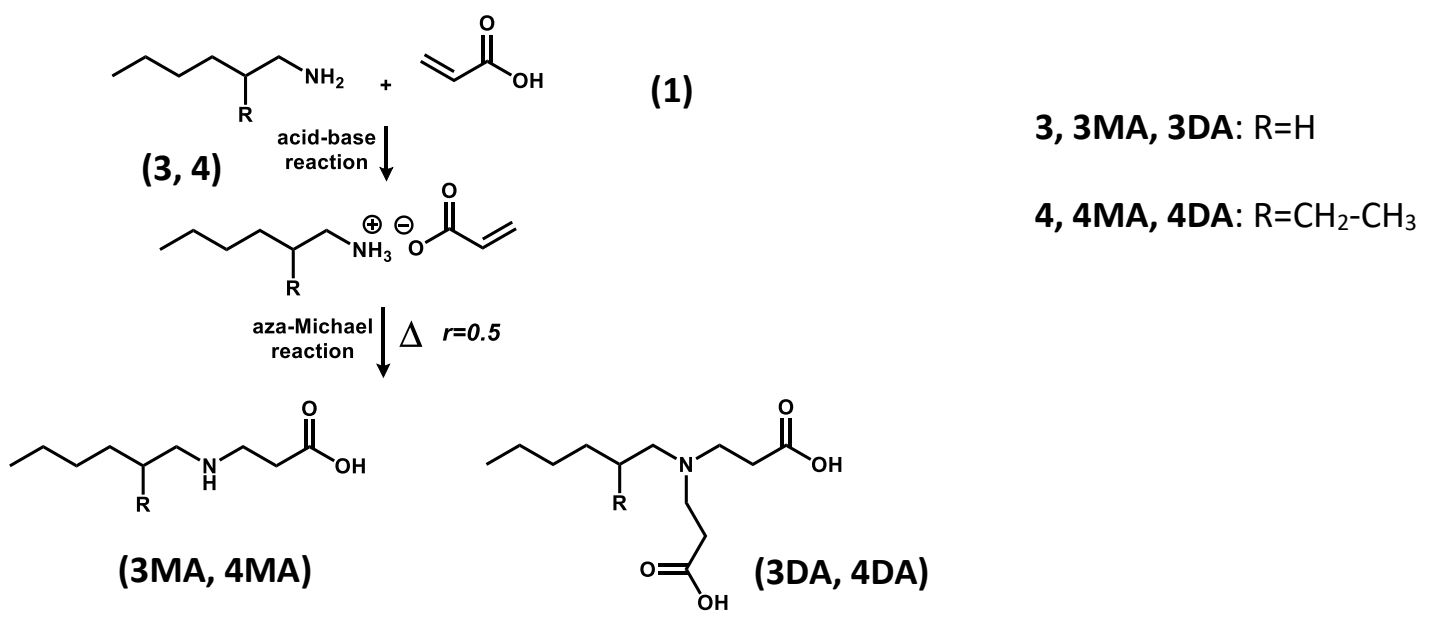

B)

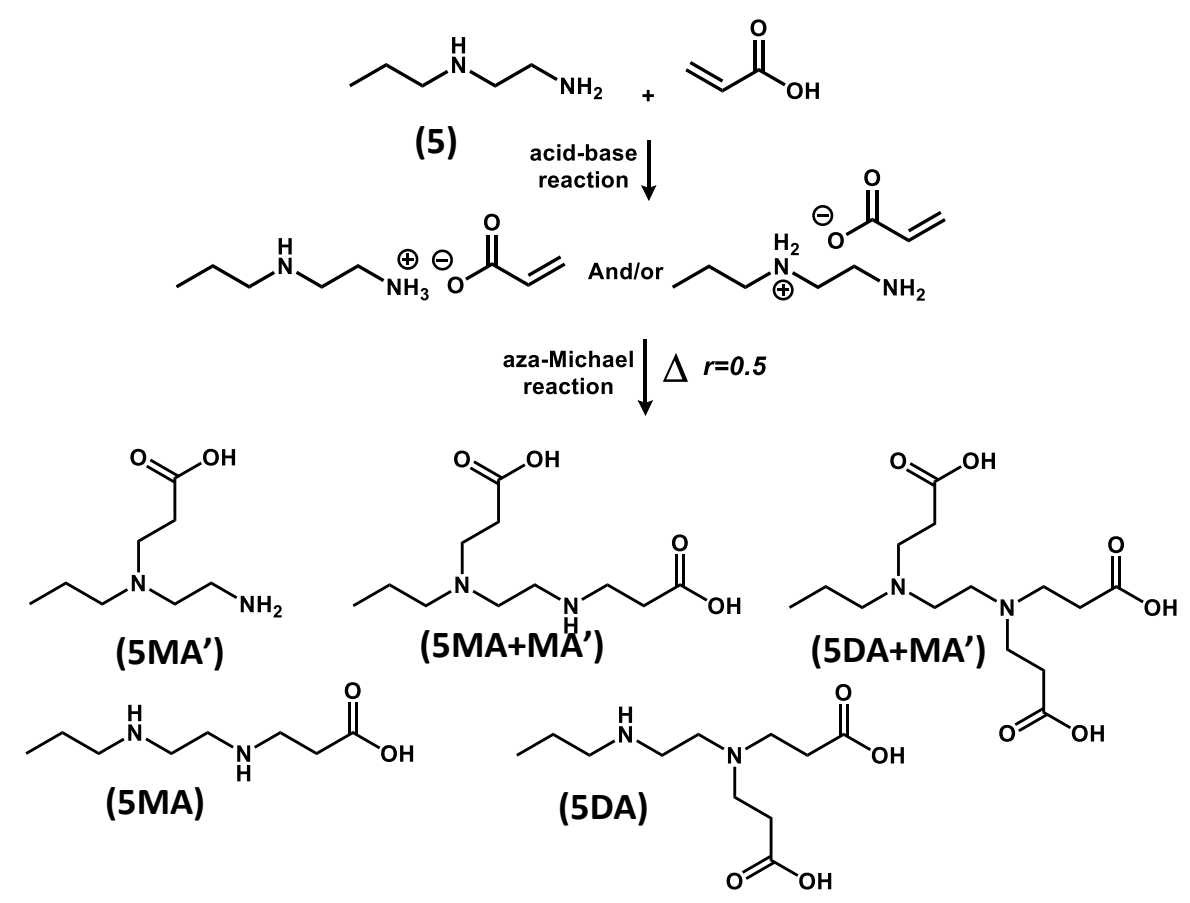

C)

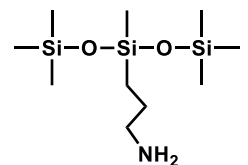

(6)

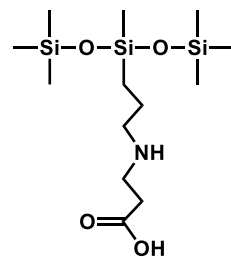

(6MA)<smiles>C[Si](C)(C)O[Si](C)(CCN(CC(=O)O)CC(=O)O)O[Si](C)(C)C</smiles>

(6DA)

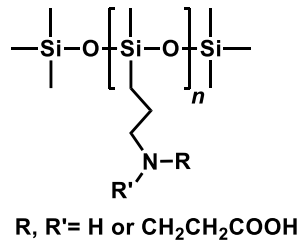

(Oligosiloxane)

$$
\mathrm{n}=\mathbf{2} \text { or } 3
$$

Figure 1: A) Set of reactions of acrylic acid (1) with hexylamine (3) and 2-ethylhexylamine (4) leading to the formation of mono-adducts (3MA, 4MA) and di-adducts (3DA, 4DA) $\left(50^{\circ} \mathrm{C}, 120 \mathrm{~h}, r=0.5\right)$; B) Set of reactions of acrylic acid (1) with N-propylethyelendiamine (5) leading to the formation of different adducts $\left(50^{\circ} \mathrm{C}, 48 \mathrm{~h}\right)$. C) Structures observed in the Michael product obtained from the addition of 3-aminopropylmethyl bis(trimethylsiloxy)silane (6) to (1) at $50^{\circ} \mathrm{C}$. 

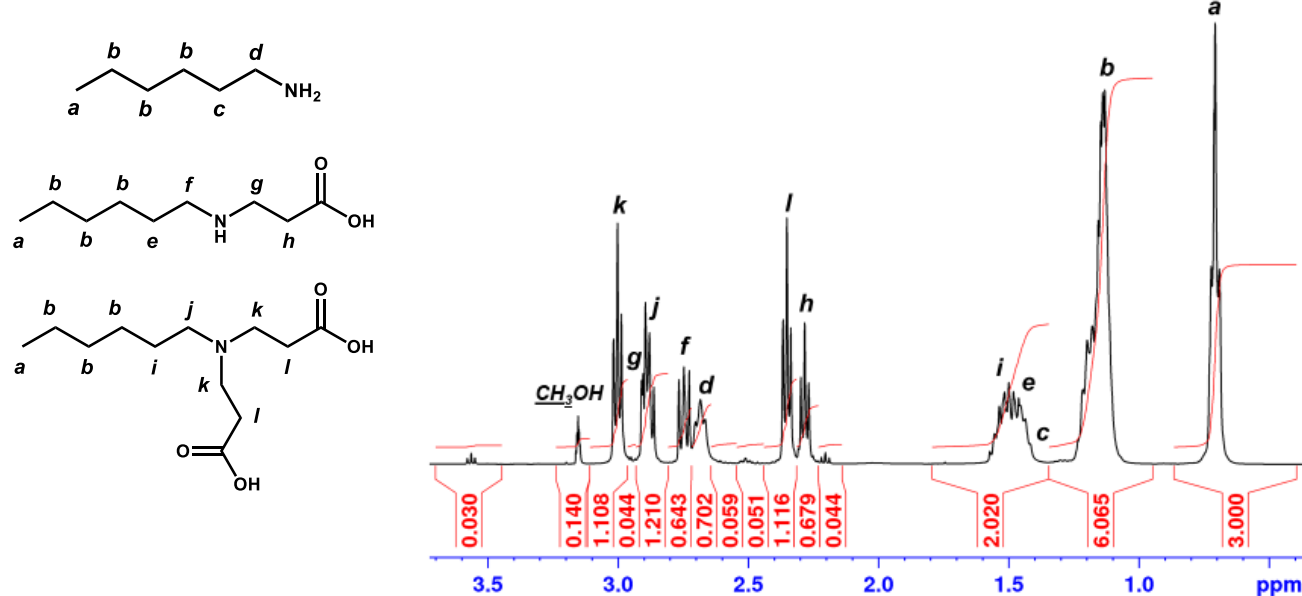

Figure 2: ${ }^{1} \mathrm{H}$ NMR analysis of products obtained from the Michael addition of acrylic acid (1) to hexylamine (3) and peak assignment for each detected molecules (99\% conversion in acrylic acid, $r=0.5$ ). Michael addition to a 2-carboxyethylacrylate impurity in the monomer is also present (see SI.2).

By applying a similar method (SI.4), the composition was also determined for the product obtained from the reaction of 2-ethylhexylamine (4) and acrylic acid (1) under equivalent conditions. This molecule was used to evaluate the impact of steric hindrance brought by the lateral ethyl group on amine reactivity. The data listed in Table 1 point out the high content of mono-adducts MA (35 to 44 mol-\%) as compared to di-adduct DA (24 to 29 mol- $\%$ ) but also the presence of residual primary amine. As a matter of comparison, when propylamine was used with butyl acrylate as Michael acceptor (bulk, $50^{\circ} \mathrm{C}, r=0.5$ ), the major product was the corresponding MA (71 mol\%) with obviously much lower content in residual primary amine (15 mol\%) and DA (14 mol\%). This suggests that the use of acrylic acid affects the selectivity of the aza-Michael reaction towards the primary amine, albeit in bulk conditions.[15] As stated in the introduction, in the literature, very few articles have dealt with the orientation of the azaMichael reaction of a primary amine with acrylic acid in the presence of polar protic solvents and no general trends could be obtained from these data where complex amines and different catalytic means were used. 
Finally, we also looked at the reaction of N-propylethylenediamine (5) that is a model molecule of telechelic silicone diamines used later in this study (see structure and reaction in Figure 1B). Conversions were quantitative after less than 2 days here, whatever the initial ratio $r$. The content of mono and di-adducts on these complex structures could not be deduced from ${ }^{1} \mathrm{H}$ NRM spectra, but showed massifs specific of all types of species show off here again (see SI.5).

To summarize, primary amine, mono-adducts and di-adducts, are all present in significant amounts when reacting acrylic acid with simple organic amines, whatever the environment of the amino groups.

\subsection{Application to aminofunctional silicones}

The aza-Michael reaction was then studied using a simple oligosiloxane molecule, i.e. 3-aminopropylmethyl bis(trimethylsiloxy)silane (6) with acrylic acid (1) at a molar ratio $r$ of 0.5 (Figure 1B). Different temperatures were tested to adapt the time of reaction and possibly avoid a degradation of $\mathrm{Si}-\mathrm{O}$ bonds and/or redistribution reactions due to the presence of acid. Kinetic data were obtained by monitoring the reaction course by ${ }^{1} \mathrm{H}$ NMR spectroscopy, and the conversion calculated from the disappearance of acrylic double bonds (Figure 3; see also SI.6). The reaction rate was logically affected by temperature. At $25^{\circ} \mathrm{C}$, a slow reaction proceeded leading to moderate conversion $(66 \%)$ even after 3 weeks. When the reaction temperature was set to $50^{\circ} \mathrm{C}$, the reaction rate was greatly improved. After $48 \mathrm{~h}$, a conversion of $94 \%$ was reached, almost four times higher than the conversion obtained at $25^{\circ} \mathrm{C}(24 \%)$. When further increasing the temperature to $70^{\circ} \mathrm{C}$, the reaction rate was enhanced even more and full conversion was obtained after $48 \mathrm{~h}$. 


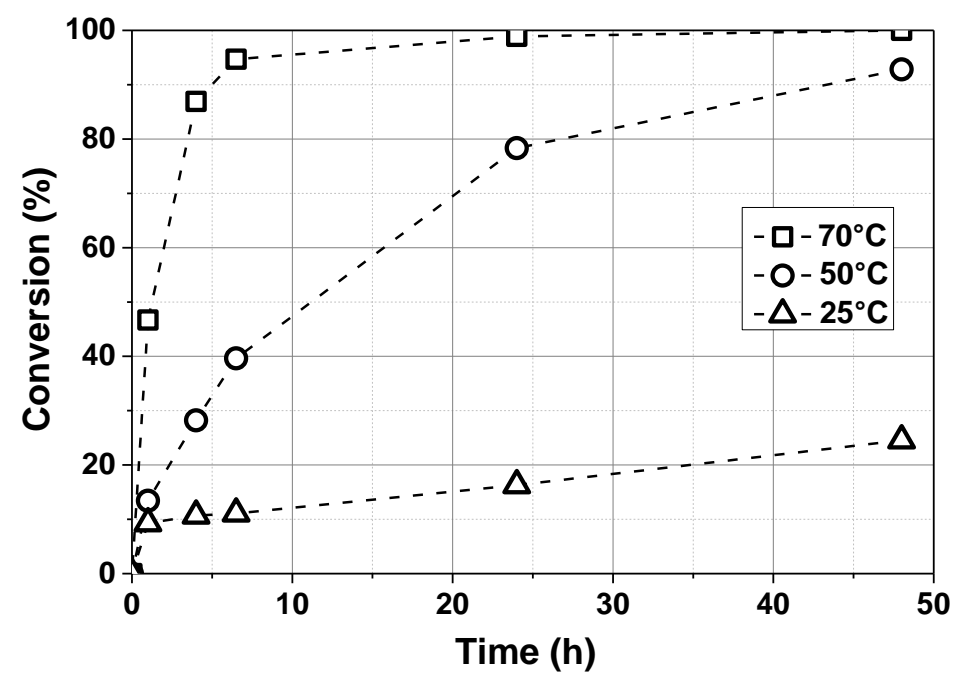

Figure 3: Influence of the temperature on the conversion of acrylic functions over time in an azaMichael reaction of 3-aminopropylmethyl bis(trimethylsiloxy)silane (6) with acrylic acid (1) $(r=0.5)$.

Unfortunately, in all these reactions, neither ${ }^{1} \mathrm{H}$ nor ${ }^{13} \mathrm{C}$ NMR analyses were capable of providing the exact structure of the Michael adducts that were formed. Both an overlap of peaks in the ${ }^{1} \mathrm{H}$ NMR spectra and a rather poor resolution due to limited ionic pair solvation in chloroform-d could explain such difficulty The products were more soluble in a deuterated chloroform/methanol solution (75/25 mol-\%) but degradation by methanolysis occurred rather fast during the analyses. The only information from the ${ }^{13} \mathrm{C}$ NMR spectrum was the presence of several peaks from 0.5 to $2.5 \mathrm{ppm}$, corresponding to carbon signals of methyl groups attached to silicon atoms with various atomic environments. ${ }^{29} \mathrm{Si}$ NMR spectra revealed clusters of peaks for trimethylsiloxane (6 to $9 \mathrm{ppm})$ and methylalkylsiloxane (-21.5 to $-24.5 \mathrm{ppm})$ units, suggesting some chemical rearrangement of the siloxane backbone via redistribution and the formation of oligosiloxanes [24] with larger degrees of polymerization (typically $n=2$ and 3) (SI.6).

This hypothesis was also supported by electrospray ionization mass analyses (ESI): residual amine-functional organosiloxane $(\mathbf{6}$, e.m.=279.15), as well as the expected mono- $(\mathbf{6 M A}$, e.m.=351.17) and di- $(\mathbf{6 D A}$, e.m.=423.19) adducts showed up in the spectrum, together with longer oligosiloxanes (SI.7). Based on the ${ }^{1} \mathrm{H}$ NMR, a following composition with $22 \%, 35 \%$ 
and $43 \%$ of MA, DA and primary amine was estimated, respectively.(Figure 1B and SI.6). Oligomers of $n=2,3$ were estimated around $5 \%$ based on the decrease of methyl peak integration of M groups, so considered as negligible here. On the other hand, hexamethyldisiloxane, that would logically form as well here, was not observed by NMR; we reckon that the long reaction time at $50^{\circ} \mathrm{C}$, could easily leads to its evaporation.

The aza-Michael reaction of acrylic acid (1) to five different amine-functional polydimethylsiloxanes (PDMSs) was finally carried out. Two PDMSs functionalized with 3aminopropyl groups, at each extremity (telechelic polymers) (7) or in a side position (grafted polymers) (8), as well as three other PDMSs $(\mathbf{9}, \mathbf{1 0}, \mathbf{1 1})$ containing N-(2-aminoethyl)-3aminopropylmethylsiloxane units (Table 2) were selected here. The reaction was tested at $50^{\circ} \mathrm{C}$ according to the protocol described above and ${ }^{1} \mathrm{H}$ and ${ }^{13} \mathrm{C}$ NMR analyses were directly conducted on the raw mixture. Despite a poor peak resolution, it was observed that acrylic acid reacted over time, as the signal of vinylic protons (5.5-6.5 ppm) almost disappeared after $72 \mathrm{~h}$ (see SI.8 experimental data for PDMS 7, 10 and 11). New peaks around 2.5-3.5 ppm were assigned to protons of methylene groups bearing hetero-atoms in an $\alpha$-position highlighting the formation of aza-Michael products, even if their exact assignment was not possible.

We could also confirm, as before, that radical polymerization of acrylic acid did not occur, regardless of the starting PDMS. To prove this fact beyond a doubt, a blank test monitored by ${ }^{1} \mathrm{H}$ NMR analyses (SI.9) was performed by mixing acrylic acid with a non-reactive silicone oil under the operating conditions used to achieve the reaction: the spectrum remained unchanged over $110 \mathrm{~h}$. From these results, it is possible to conclude that the aza-Michael reaction of acrylic acid and aminosilicones could be efficiently carried out in bulk by simply mixing both reactants at $50^{\circ} \mathrm{C}$ for a sufficient time so as to generate a mixture of new zwitterionic polymers (Figure 4). As stated before, catalyst or solvent were not used to avoid backbitting redistribution 
reactions and purification steps as well as higher temperature than $70^{\circ} \mathrm{C}$ was not used to avoid amidation side reactions.

Table 2: Chemical structures of the amine-functional polydimethylsiloxanes selected in this study

\begin{tabular}{ccccc}
\hline PDMS & $\begin{array}{c}\text { Nature of } \\
\text { aminated } \\
\text { unit }\end{array}$ & $\begin{array}{c}\text { Aminated unit } \\
\text { contents } \\
(\mathrm{mol}-\%)\end{array}$ & $\begin{array}{c}\overline{\mathrm{M}}_{\mathrm{w}}{ }^{(4)} \\
\left(\mathrm{g} \cdot \mathrm{mol}^{-1}\right)\end{array}$ & $\begin{array}{c}\overline{\mathrm{M}}_{n} \\
\left(\mathrm{~g} \cdot \mathrm{mol}^{-1}\right)\end{array}$ \\
\hline $\mathbf{7}$ & $\mathrm{M}^{\text {amp (1) }}$ & 5 & 3,000 & $2,600^{(5)}$ \\
$\mathbf{8}$ & $\mathrm{D}^{\text {amp (2) }}$ & 10 & $2,000-3,000$ & $2,750^{(5)}$ \\
$\mathbf{9}$ & $\mathrm{D}^{\text {aeamp (3) }}$ & 3 & 20,000 & $20,000^{(6)}$ \\
$\mathbf{1 0}$ & $\mathrm{D}^{\text {aeamp (3) }}$ & 1 & 28,000 & $38,500^{(5)}$ \\
$\mathbf{1 1}$ & $\mathrm{D}^{\text {aeamp (3) }}$ & 0.5 & $\mathrm{~N} . \mathrm{A}$. & $62,000^{(6)}$ \\
\hline
\end{tabular}

${ }^{(1)} \mathrm{M}^{\mathrm{amp}}$ (end group): 3-aminopropyldimethylsiloxane unit

(2) $\mathrm{D}^{\text {amp }}$ (side group): 3-aminopropylmethylsiloxane unit

(3) $\mathrm{D}^{\text {aeamp }}$ (side group): $\mathrm{N}$-(2-aminoethyl)-3-aminopropylmethylsiloxane unit

(4) Given by the supplier.

(5) Obtained by ${ }^{29} \mathrm{Si}$ NMR peaks integration

(6) Estimated from the complex viscosity of chains put in reaction with (2) (See SI.10 for details of calculation).

\subsection{Thermal properties}

As expected, only one $\mathrm{Tg}$ was detected at around $-123^{\circ} \mathrm{C}$ for bis-(3-aminopropyl)-terminated PDMS (7) and neither a melting nor crystallization transitions appeared, in agreement with its low molecular weight (data not shown). In comparison, the product obtained after aza-Michael reaction presented a first $\mathrm{Tg}$ at $-116{ }^{\circ} \mathrm{C}$ and an additional glass transition at $-40{ }^{\circ} \mathrm{C}$ (see Figure SI.11A). These corresponded to transitions taking place in the PDMS matrix, and in the ionic domains constituting a second separated phase, respectively. Huang et al. described a similar behavior for polysiloxane ionomers bearing quarternary ammonium groups, exhibiting two glass transition temperatures: one at $-121^{\circ} \mathrm{C}$ (polysiloxane matrix) and the other at $-20^{\circ} \mathrm{C}$ (polar phase) [25]. In the literature, the size of the ionic clusters has been tracked at 
the nanoscale. The transparency of the products described herein (vide infra) makes us believe that the ionic domains were much smaller than $50 \mathrm{~nm}$ as in the case of an ionic polyisoprene elastomer [26].

A)

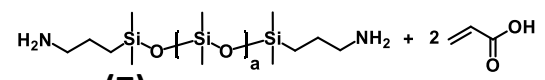

(7) Acid-base
reaction<smiles>C=CC(=O)O[Na]</smiles>
Aza-Michael
reaction

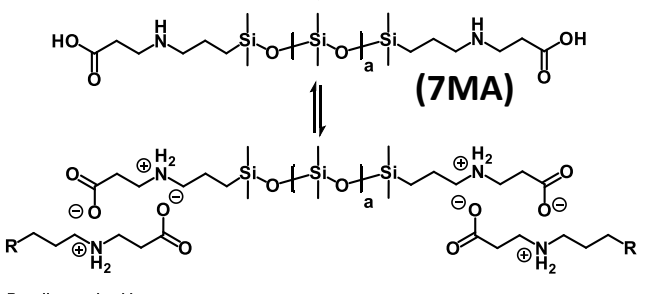

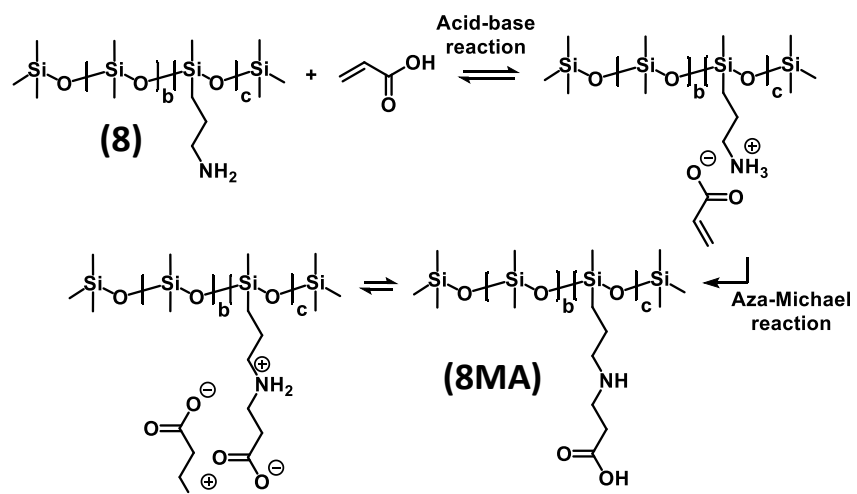

B)<smiles>C[Si](C)(O)CCNCN</smiles>

Primary/ Secondary amines<smiles>C[Si](O)(O)CCN(CCN)CCC(=O)O</smiles>

Primary/tertiary amines

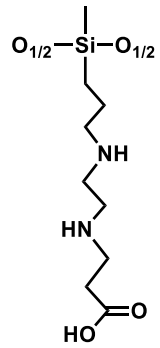

Secondary amines

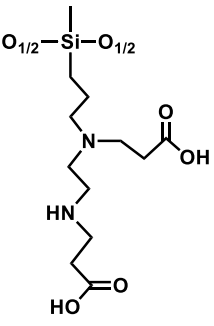

Secondary/tertiary amines<smiles>C[Si](C)(O)CCN(CCC(=O)O)CCN(CCC(=O)O)CCC(=O)O</smiles>

Tertiary amines

Figure 4: A) Synthesis of zwitterionic-PDMS by aza-Michael reaction (for better clarity, only the monoadducts are represented). B) Possible structures stemming from the aza-Michael reaction of acrylic acid (1) with $\mathrm{N}$-(2-aminoethyl)-3-aminopropylmethylsiloxane units.

TGA experiments were also performed with the same amino-functional silicone 7 and its zwitterionic analogue obtained from the mixture (1) + (7) (see Figure SI.11B). Both silicones were highly sensitive to temperature degradation, with $50 \mathrm{wt} . \%$ losses observed at 380 and $430^{\circ} \mathrm{C}$, respectively. The presence of a relatively high content (> $\left.5 \mathrm{wt}-\%\right)$ of trapped water was also detected between 100 and $150^{\circ} \mathrm{C}$ for both components, confirming the polar character of functional groups. 


\subsection{Rheological behavior of zwitterionic PDMS}

\subsubsection{Correlation between chemistry and rheological properties}

The occurrence of an aza-Michael reaction was supported by the drastic change in viscosity (measured at $25^{\circ} \mathrm{C}$ ) dependent on the progress of the reaction and initial reagents (see Figure 5A). Hereto, all the reactive systems maintained a Newtonian behavior but seemed to be quite sensitive to the effect of reaction times. For instance, the starting amino-functional PDMS 7 naturally comes as very low and Newtonian viscosity oil (around 0.06 Pa.s for $r=0.5$ ). When acrylic acid (1) was put into play, a 20-fold increase in viscosity was observed directly after mixing $(\mathrm{t}=0)$. Such an increase confirmed the instantaneous acid-base reaction that naturally takes place between the carboxylic acid groups and the amine groups of the functional PDMS. A comparable extent of viscosity increase was observed when using propionic acid (2)

(instead of acrylic acid (1)) with PDMS 7. But the most interesting result was the 3000-fold increase in viscosity observed after 72 hours at $50^{\circ} \mathrm{C}$ for the system involving acrylic acid (178 Pa.s), whereas the one involving propionic acid showed a constant viscosity after the same time (0.66 Pa.s). Such an increase in viscosity for the mixture of $(\mathbf{1})+(\mathbf{7})$ was correlated with the concomitant aza-Michael reaction, supramolecular assembly of the thus-created zwitterionic moieties and phase-separation of highly polar ionic clusters.

\subsubsection{Linear viscoelasticity of zwitterionic materials prepared from PDMS 7}

A set of zwitterionic materials was prepared by modifying the amino-functional polydimethylsiloxane $\mathbf{8}$ via aza-Michael reactions with acrylic acid (1) in various contents (molar ratios ranging from $r=0.13$ to $r=1$ ). We recorded the complex viscosity of each material as a function of the frequency at room temperature. Figure 5B clearly highlights that the complex viscosity increased even in the presence of an excess of carboxylic acid groups. 
A

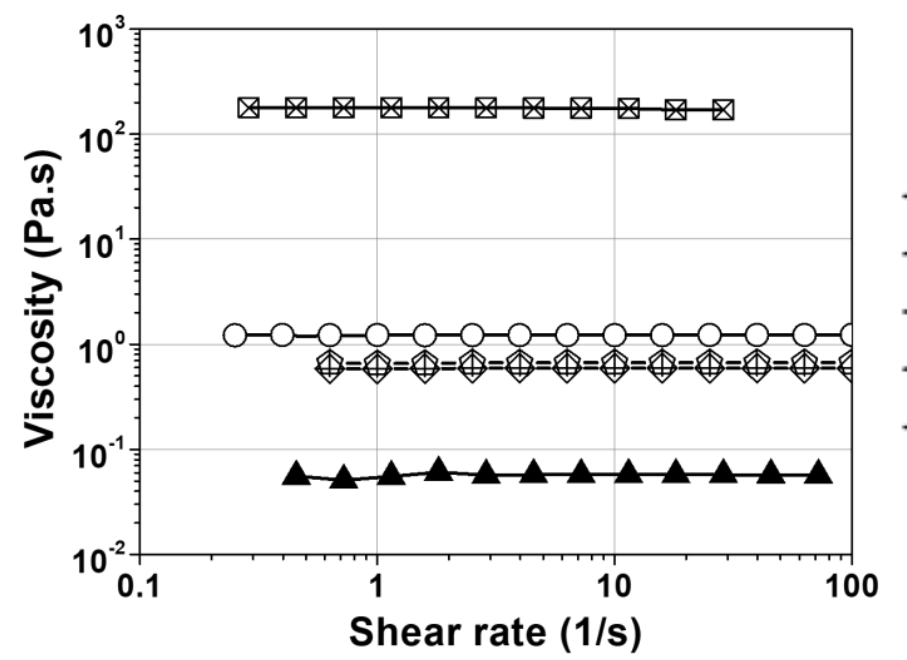

B)

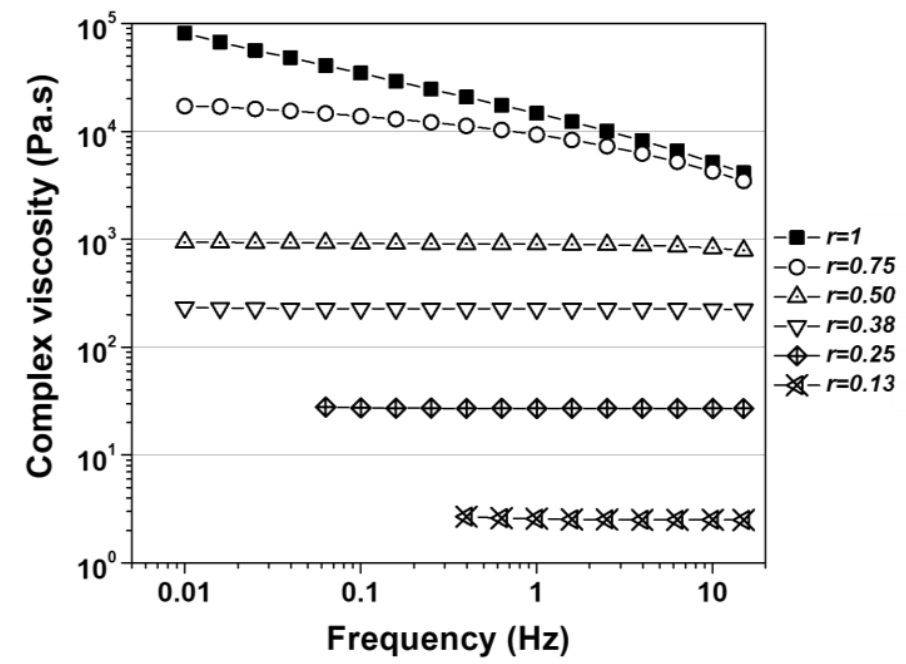

Figure 5: A) Apparent viscosity versus nominal shear rate measured under steady shear mode in cone-plate rheometer up to different stages of reaction between bis-(3-aminopropyl)-terminated PDMS (7)/acrylic acid (1) or propionic acid (2) $(r=0.5)$. B) Complex viscosity modulus of zwitterionic silicone with various zwitterionic contents obtained from an aza-Michael reaction of acrylic acid (1) with PDMS $(\mathbf{8})\left(r=0.13-1,50^{\circ} \mathrm{C}\right)$ 
More precisely, at low acrylic acid content $(r \leq 0.5)$, a Newtonian behavior with a complex viscosity plateau was observed over the entire frequency range. This is the signature of a viscous fluid in the terminal flow regime [27]. It is interesting to note that with only a 2-fold increase of the molar ratio ( $r=0.25$ to 0.5 ), a 30 -fold increase of the complex viscosity modulus was observed. Finally, using an excess of carboxylic acid groups $(r=0.75-1)$, the behavior of the product changed completely: the complex viscosity decreased at high frequency and no plateau value was reached for the product prepared at $r=1$ in the measured frequency range attesting a frequency-thinning behavior (equivalent to shear thinning for Non-Newtonian fluids).

The reflectance of the complexation-decomplexation kinetics in the macroscopic dynamics of supramolecular polymer networks is not only a useful tool but it is also interesting for fundamental polymer physics. For this purpose, the viscoelastic data have been represented by plotting the storage and loss moduli as functions of frequency. For all the studied materials, the loss modulus $G$ "' remained higher than the storage modulus $G$ '. Indeed, a liquid-solid like rheological behavior is assumed in supramolecular polymers. An universal time-concentration superposition plot was then created by applying vertical and horizontal shifting/translation factors (SI.12), and the obtained master curve was fitted with a Rouse model (Figure 6A) [28]. When $r$ remained below 0.5 , a good superimposition of both the storage and loss moduli and the Rouse fits is observed, with G' and G' curves reaching slope values of 2 and 1, respectively. These zwitterionic silicones thus follows the same classical behavior as of non-entangled polymers with terminal relaxation times $[29,30]$. However, with an excess of carboxylic acid groups ( $r=0.75$ or 1$)$, both the storage and loss moduli moved away from the Rouse model $[27,30]$. In that case, the dynamics of the chains obey two different characteristic timescales: one is the timescale of formation and breakage of supramolecular associations, while the other is the timescale for the relaxation of chains or chain segments as captured by Rouse model, which is still applying at higher angular frequencies. The obtained results corroborate those of 
Mursean et al. [31] regarding the dynamic of UPy-functionalized poly(THF). Indeed, the authors found that supramolecular lengthening and entanglement of associating polymer chains follow the expected Rouse-type motion.

Furthermore, the phase angle $\left(\delta=\tan ^{-1}\left(G^{\prime \prime} / G^{\prime}\right)\right)$ of the measured rheological data was also plotted against the corresponding absolute value complex modulus $G^{*}$ according to the van Gurp and Palmen (vGP) method [32,33] (Figure 6A, inset). This approach, which normally allows verifying the validity of the time temperature superposition (TTS), was used here to provides insight in the thermorheological simplicity or complexity of the polymer chains following a linear viscoelastic behavior [34]. Here, the $\delta$ values at low $G^{*}$ clearly depends on the ratio $r$. The Van-Gurp-Palmen plots shows a linear decrease in phase angle values when increasing the $r$ molar ratios. For $r$ values below $0.5, \delta$ approached $90^{\circ}$ indicating a dominant viscous state. For ratios $r$ equal to 0.75 or 1 , the $\delta$ values decrease when increasing the acrylic acid content, which is consistent with an enhancement of the elasticity of these samples. More interestingly, both systems with $r$ values of 0.75 and 1 exhibited the classical shape of a viscous polymer; the phase angle presenting a relatively long plateau before dropping to the same value of an apparent plateau modulus $G_{0}{ }^{*}$ (when $\delta \rightarrow 0$ ) assuming that the obtained supramacromolecules displayed the same thermorheological properties regardless of their complexation-decomplexation kinetics. In summary, the deviation of the viscoelastic properties can once again be explained by the presence of ionic domains inside the product; the number of domains and the extent of phase separation certainly strengthen when $r$ increases, probably related to generation of di-adducts. 
A)

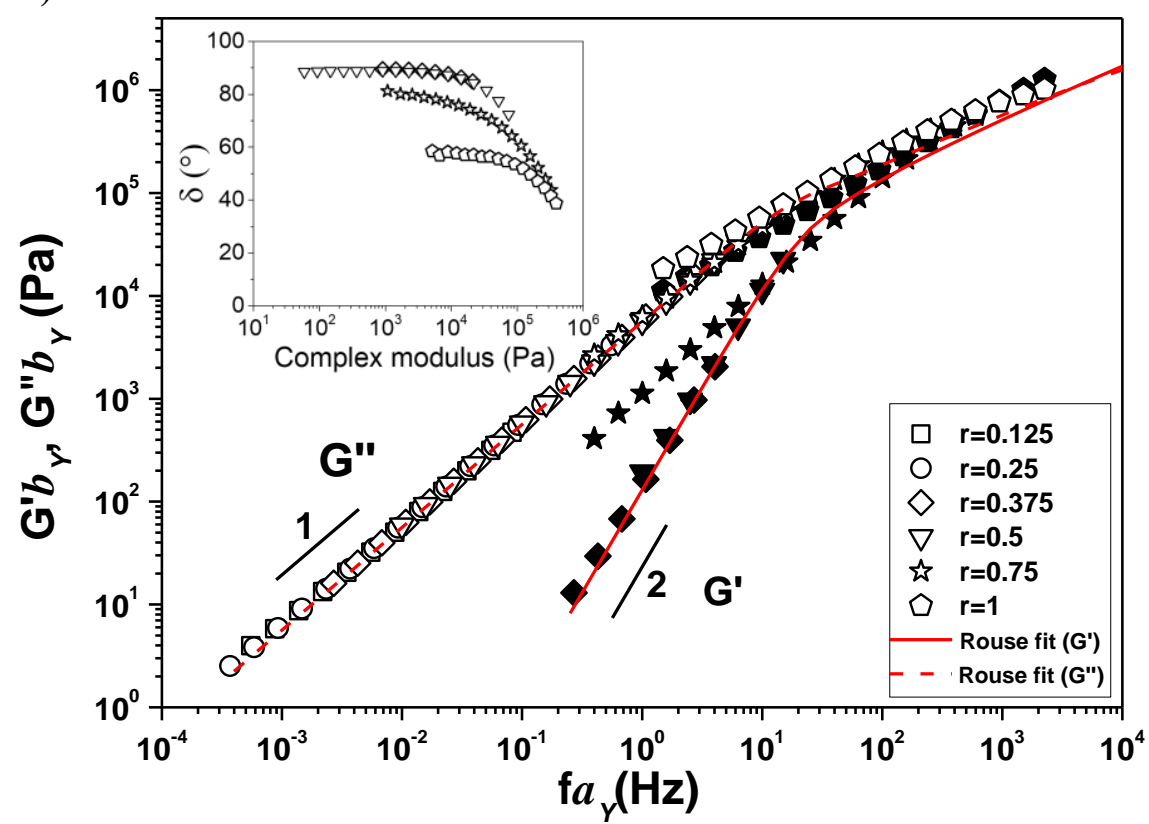

B)

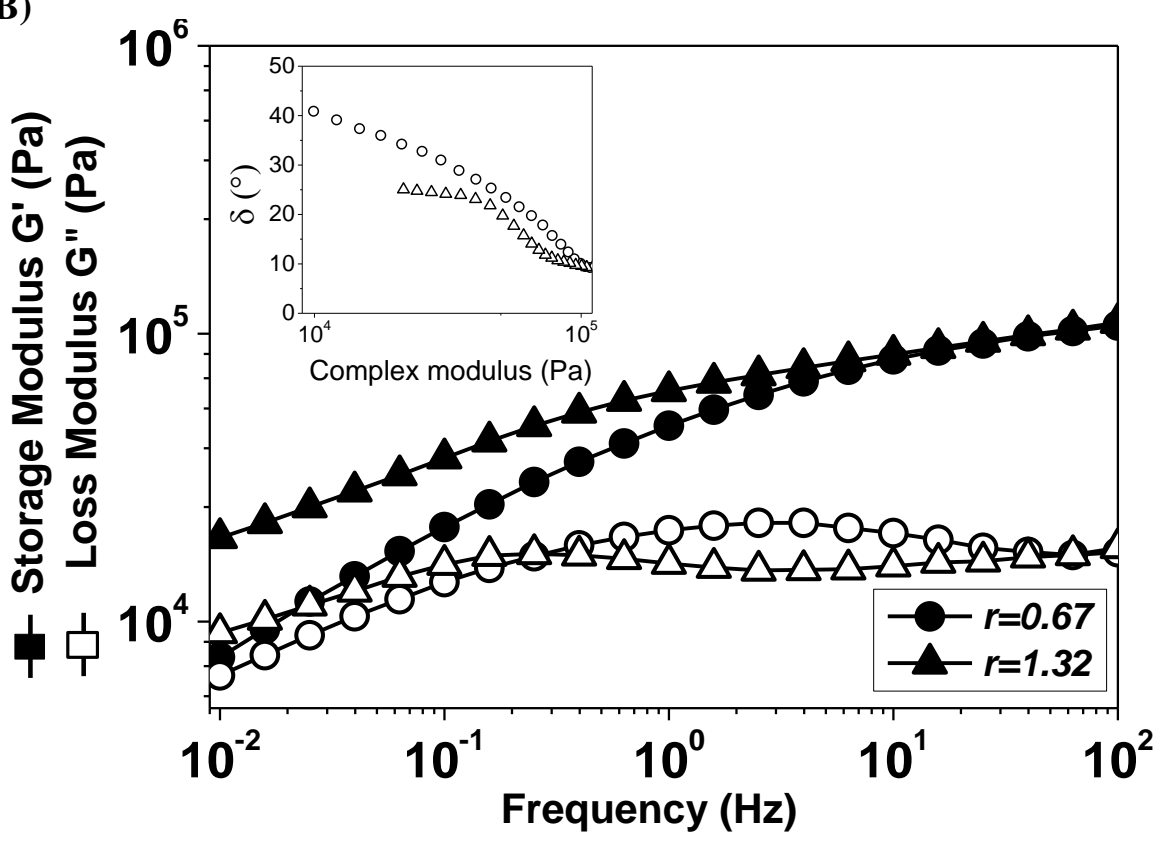

Figure 6: (A) Viscoelastic properties of zwitterionic silicones with various zwitterionic contents obtained from an aza-Michael reaction of (1) with PDMS (8) $\left(r=0.13-2,25^{\circ} \mathrm{C}\right.$, master curve). B) Storage $\left(G^{\prime}\right)$ and loss $\left(G^{\prime \prime}\right)$ moduli versus frequency of zwitterionic silicone based on N-(2-aminoethyl)-3aminopropylmethylsiloxane-dimethylsiloxane copolymer $(9)$ at two ratios $r\left(25^{\circ} \mathrm{C}\right)$. Insets: Representative vGP curves (see text for details). 


\subsubsection{Toward zwitterionic elastomers}

Zwitterionic materials were tentatively prepared by a reaction of acrylic acid (1) and silicone (8) exhibiting a low molecular weight and bearing 3-aminopropyl groups in a lateral position (see Table 2, $r=0.13$ to 1 ). Despite the relatively high concentration of initial amine functions, no elastomer material was obtained, indicating that physical crosslinking did not occur here even with acrylic acid in excess. Additional investigations were performed with two different PDMSs $(9,10)$ at $r=0.67$, both of which had a higher molecular weight and more complex $\mathrm{N}$ (2-aminoethyl)-3-aminopropyl side functions. This time, the aza-Michael reaction with acrylic acid produced soft elastomers. Meanwhile, the viscoelastic data gathered for PDMS (9) (Figure 6B) clearly showed that the storage modulus was superior to the loss modulus over the explored frequency domain (from 100 to $0.01 \mathrm{~Hz}$ ). Furthermore the method of van Gurp and Palmen (vGP) was applied to our results (Figure 6B, inset) and the low level of $\delta$ values below $60^{\circ}$ (about $40^{\circ}$ and $25^{\circ}$ respectively for $\mathrm{r}=0.67$ and 1.32 at lowered frequencies) confirmed the solidlike character with dominant elastic behavior of the studied zwiterrionic materials. Once again, both formulations converge at the same extrapolated plateau modulus $G_{0}{ }^{*}$. Similar results were obtained with PDMS (10) and are shown in supplementary information (Figure SI.12.2).

The mechanical properties of the previously obtained elastomers remained too weak to be measured. A final PDMS (11), also bearing N-(2-aminoethyl)-3-aminopropyl lateral functions but with a higher molecular weight was tested (Table 2) at $\mathrm{r}=0.67 .[35]$ The elastomeric character of these transparent materials is obviously derived from the development of numerous ionic interactions between the silicone chains thereby promoting a physical network of polar clusters (Figure 7A) visualized by TEM (Figure 7B). PDMS with N-(2-aminoethyl)3-aminopropyl functions favored a high degree of physical crosslinking because of the increased probability of obtaining two or more interaction points especially with silicone units 
bearing secondary and tertiary amines or exclusively tertiary amines on side groups (see Figure

4B).

A)
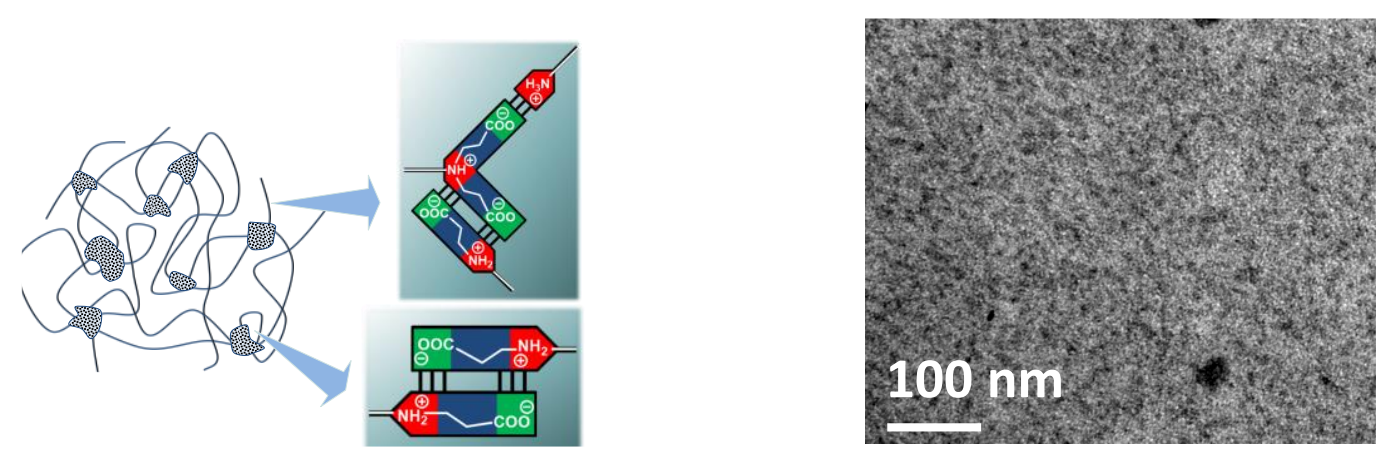

D)

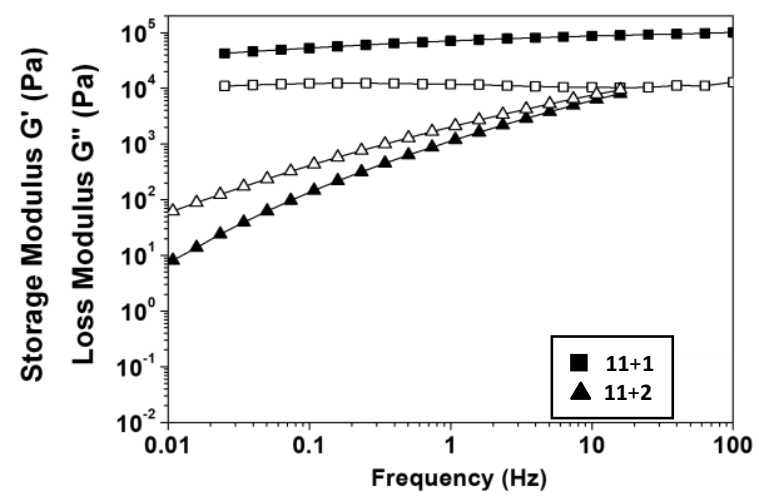

E)

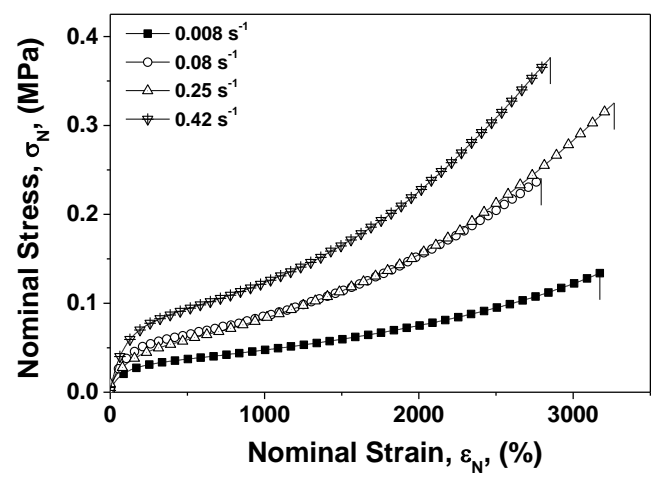

Figure 7: Properties of materials prepared by reaction of silicone (11) and acrylic acid (r=0.67) (1) or propionic acid (2) at $50^{\circ} \mathrm{C}$ during 1 week. A) Scheme of phase demixtion, showing polar cluster formation through ionic pairing; B) TEM image of ultrathin sections of final silicone (11+1) showing clustering of ionic domains; C) Storage (G') and loss (G', moduli versus frequency of final materials $(\mathbf{1 1}+\mathbf{1})$ and $(\mathbf{1 1}+\mathbf{2})\left(25^{\circ} \mathrm{C}, 0.25-1 \%\right.$ strain $)$; D) Nominal stress-strain profiles for zwitterionic silicone product $(11+1)$ in uniaxial tension at various strain rates.

The storage and loss moduli profile of this silicone (11) after reaction with acrylic acid (1) clearly showed that the elastic modulus $(\sim 50-100 \mathrm{kPa})$ was higher than the loss modulus $(10 \mathrm{kPa})$ on the whole frequency range. As a matter of comparison, profiles observed with using propionic acid (2) instead of (1) confirmed that a viscoelastic liquid was obtained as expected (Figure 7C). In addition, mechanical properties of the zwitterionic silicone by uniaxial tensile tests showed a high dependency on the strain rate. An increase of the strain rate led to higher stress levels and to slightly lower elongation at break. (Figure 7D) This strain rate dependency has never been shown in hyper-elastic materials such as chemically cross-linked silicone elastomers filled with silica, obtained by classical chemical pathways [36]. In physically cross- 
linked elastomers such as zwitterionomers reported by Graiver et al. [11], such strain-rate dependency was clearly observed for a product with a low content of zwitterionic groups (1-2 mol-\%) and for higher molar content (5-10 mol-\%), to a lesser extent. Thus, physical interactions in this zwitterionic silicone as well as its high molar mass greatly impacted the mechanical properties of the product. At low strain rate, the network dynamic allowed the clusters to break and re-form, while this was not the case at higher strain rate, at least within the experimental timescale. Note that even if the tensile strength is of moderate value (typically $0.5 \mathrm{MPa}$ ), remarkable elongation was noticed (up to $3000 \%$ ).

\section{Conclusion}

This article detailed the synthesis of novel zwitterionic silicones obtained from aza-Michael reaction between acrylic acid and various amine-functional polydimethylsiloxanes. These reactions proceeded according to a two-step protocol without any solvent, including a first step of mixing reactants at room temperature to overpass the heat release due to the acid-base reaction, and then a long temperature ramp step to let the aza-Michael reaction proceed until completion. The protocol was first validated on a model reaction with three alkylamines and then efficiently transferred to amino silicone reagents.

Thermal characterizations as well as steady flow and linear rheological analyses confirmed the phase separation between the silicone matrix and clusters of the hydrophilic zwitterionic groups resulting from aza-Michael reactions. Moreover, both drastic increases in viscosity and

moduli corroborated the formation of ionic clusters from supramolecular assemblies. More specifically, the Small Amplitude Oscillatory Shear (SAOS) investigations with the original vGP (Van-Gurp-Palmen) plots turned out to be an elegant and suitable probe of the structureviscoelasticity property relationships of the zwitterionic supra-macrolecular materials. Finally, the combination of two structural parameters, namely lateral N-(2-aminoethyl)-3-aminopropyl 
functions and high molecular weight, led to silicone elastomers with solid-like behavior and measurable mechanical properties, opening the way to generate filler-free soft silicone elastomers.

\section{Acknowledgement}

The authors gratefully acknowledge the NMR Polymer Center at "institute de Chimie de Lyon" (FR 3023) for their access to NMR facilities and helpful comments. This work was supported by ANRT CIFRE (contract \#:0717/2012).

\section{References}

[1] Yang L., Tan X., Wang Z., Zhang X. Chem. Rev. 2015, 115, 7196.

[2] Winter A., Hager M. D., Schubert U.S. 'Polymer Science: A Comprehensive Reference', Vol. 5, 2012, pp. 269-310.

[3] Faul C. F. J., Antonietti M. Adv. Mater. 2003, 15, 673.

[4] Klok H.-A., Rebrov E., Muzafarov A. M., Michelberger W., Möller M. J. Polym. Sci. Polym. Phys. 1999, 37, 485.

[5] Saxene A., Sarkar A., Tiwari S. Silicone ionomer composition. WO2013103496. 2013. Momentive Performance Materials.

[6] Cummings M., Horstman J. B., Norris A. W., Swier S. Use of ionomeric silicone thermoplastic elastomers in electronic devices. WO2010147759. 2010. Dow Corning Corporation.

[7] Lu H., Feng S., J. Polym. Sci. Polym. Chem. 2017, 55, 903.

[8] Graiver, D., Baer E., Litt M., Baney R. H. J. Polym. Sci. Polym. Chem. 1979, 17, 3559.

[9] Yu, X.; Nagarajan, M. R.; Li, C.; Gibson, P. E.; Cooper, S. L. J. Polym. Sci. Polym. Phys. $1985,23,2319$.

[10] Graiver, D.; Litt, M.; Baer, E. J. Polym. Sci. Polym. Chem. 1979, 17, 3573. 
[11] Graiver, D.; Litt, M.; Baer, E. J. Polym. Sci. Polym. Chem. 1979, 17, 3625.

[12] Brook M.A. 'Silicon in Organic, Organometallic and Polymer Chemistry', Wiley, New York 2000, pp. 256-258.

[13] Wang X., Wang H., Sun Y. Chem 2017, 3, 211.

[14] Genest A., Portinha D., Fleury E., Ganachaud F. Prog. Polym. Sci. 2017, 8, 61.

[15] Genest A., Binauld S., Pouget E., Ganachaud F., Fleury E., Portinha D., Polym. Chem. $2017,3,624$.

[16] Critchfield F. E., Funk G. L., Johnson J. Anal. Chem. 1956, 28, 76.

[17] Dokichev T. V., Latypova D. R., Biglova R. Z., Talipov R. F. Dokl. Chem. 2010, 430, 47.

[18] Farahani M., Antonucci J. M., Karam L. R. J. Appl. Polym. Sci. 1998, 67, 1545.

[19] Dubois L., Acher F., McCort-Tranchepain I. Synlett 2012, 23, 791.

[20] Skorik Y. Polym. Bull. 2012, 68, 1065.

[21] Sashiwa H., Yamamori N., Ichinose Y., Sunamoto J., Aiba S. Macromol. Biosci. 2003, 3, 231.

[22] Skorik Y., Pestovc A. V., Kodessc M. I., Yatluk Y. G. Carbohydr. Polym. 2012, 90, 1176.

[23] Rulev A.Y., Russ. Chem. Rev. 2011, 80, 197.

[24] Shooshtari,K. A., Van De Mark M. Polym. Prepr. 2001, 42, 458.

[25] Huang Z., Yu Y., Huang Y. J. Appl. Polym. Sci. 2002, 83, 3099.

[26] Miwa Y., Kurachi J., Kohbara Y., Kutsumizu S. ChemComm 2018, 5, 1.

[27] Ferry J. D., 'Viscoelastic properties of polymers', Wiley, New York, 1980.

[28] Stricher A., Picard L., Gabrielle B., Delebecq E., Ganachaud F., Polym. Int. 2016, 65, 713.

[29] Dealy J. M., Larson R. G. 'Structure and rheology of molten polymers: from structure to flow behavior and back again', Hanser: Munich, 2006.

[30] Zhang H., Lamnawar K., Maazouz A. Ind. Engineer. Chem. Res., 2018, 57, 8093. 
[31] Mursean A. S., Dubbeldam J. L. A., Kautz H., Monkenbusch M., Sijbesma R. P., van der Scoot P., de Jeu W. H. Phys. Rev. E, 2006, 74, 031804.

[32] van Gurp M., Palmen J. Rheol. Bull., 1998, 67, 5.

[33] Trinkle S., Walter P., Friedrich C. Rheol. Acta., 2002, 41, 103.

[34] Lu B., Lamnawar K., Maazouz A., Zhang H. Soft Matter, 2016, 12, 3252.

[35] Ganachaud F., Fleury E., Portinha De Almeida D., Genest A., Pouget E. WO2016102498. 2015. Bluestar Slicones France.

[36] Stricher A., Rinaldi R., Barrès C., Ganachaud F., Chazeau L. RSC Adv. 2015, 53713. 


\section{TOC graph}
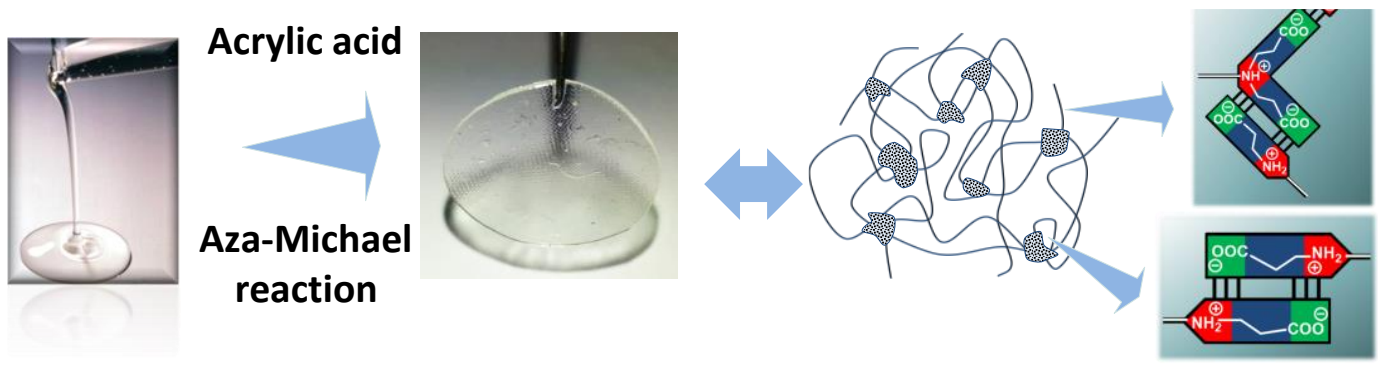

\section{Summary}

Supramolecular zwitterionic silicones were synthesized by aza-Michael reaction between acrylic acid and amine-functional polydimethylsiloxanes. The ionic pairing of thus-created zwitterionic moieties was highlighted by concomitant increase in viscosity and occurrence of phase-separation. Elastomers showing fair mechanical properties were obtained using high molar mass polymers bearing large loads of bifunctional units favoring a high degree of physical crosslinking 